\title{
Préoccupations nutritionnelles et communication de l'industrie
}

\section{Nutritional concerns and communication}

Oléagineux, Corps Gras, Lipides. Volume 7, Numéro 1, 99-100, Janvier - Février 2000, Dossier : actes des Journées Chevreul "Corps gras, nutrition et santé, questions d'actualité" (Bordeaux, Pessac)

Auteur(s) : Valérie BUSSON, Responsable des relations scientifiques, Astra Calve, 23, rue FrançoisJacob, TSA 20004, 92842 Rueil-Malmaison Cedex.

Author(s) : Valérie BUSSON

Résumé : Aujourd'hui, dans un contexte alimentaire mouvementé, guidé par des enjeux politiques et économiques d'envergure, bouleversé par les craintes croissantes des consommateurs (qu'elles soient fondées ou non) et par de véritables révolutions en termes d'offre, les industries agroalimentaires s'efforcent de proposer aux consommateurs des produits dont la mission dépasse les attentes en qualités habituellement exprimées : il s'agit de tenir compte dans le développement des produits des résultats des données scientifiques internationales, sans transiger sur la qualité et le goût des produits dont il faut rappeler qu'ils doivent être consommés par tous. Ainsi, les produits de marque sont ils reconnus par les consommateurs non seulement sur des identités d'emballage mais sur un aspect, une texture un goût définis. Modifier une formule pour actualiser sa composition en tenant compte des plus récentes données scientifiques constitue donc un véritable défi pour les développeurs qui doivent optimiser les recettes en modifiant le moins de paramètres apparents possible pour ne pas risquer de déstabiliser l'identité du produit concerné. Au-delà, il faudra que la communication du produit s'inscrive dans une réalité réglementaire, parfois contraignante, et tienne compte du niveau d'informations réel du consommateur. Pour illustrer ces propos, nous prendrons trois exemples.

\section{ARTICLE}

\section{Les acides gras essentiels (AGE)}

Les entreprises travaillant dans la fabrication des corps gras ont été les premières à communiquer dans le domaine de la nutrition. Pour sa communication nutritionnelle, le groupe Unilever et AstraCalvé, sa filiale en France, ont choisi de fonctionner par cascade et de commencer à informer et intéresser les réseaux des professionnels de la santé comprenant aussi bien les diététiciens que les médecins généralistes.

Les AGE : un bouleversement global

Au-delà des produits finis, l'ensemble des résultats émanant des recherches scientifiques sur l'acide linoléique va révolutionner le paysage alimentaire et le paysage agricole puisque l'ensemble de la France se couvre de champs de tournesol. Ce sera donc l'avènement du tournesol dans les années 60. 
Les margarines au tournesol vont pouvoir alléguer de leur garantie en AGE (acide linoléique) si la part présente en acides gras linoléiques représente au minimum $50 \%$ de l'apport global en matières grasses (ce n'est pas forcément simple car, naturellement, l'apport en linoléiques du tournesol varie en fonction des récoltes et des années) (figure). Pour maintenir l'apport au niveau de $50 \%$ et pour bénéficier de l'allégation, il faut choisir les huiles high Pufa ou compléter par du carthame.

Un peu plus tard, on assiste à l'arrivée du 18:3 qui va, dans l'opinion publique, acquérir ses lettres de noblesses avec la forte médiatisation de la Lyon Study des Pr De Lorgeryl et Renaud.

L'ère du tournesol est révolue et, pour Unilever et Astra-Calvé, toutes les margarines vont intégrer progressivement du colza dans leur formule. Ceci va poser un certain nombre de contraintes pour l'industrie :

- des contraintes techniques. Les huiles riches en 18:3 (colza) s'auto-oxydent très vite et sont moins stables que les huiles de tournesol : paramètre important dont il faut tenir compte dans le développement des produits car ceci a une action directe sur ses qualités organoleptiques ;

- des contraintes de la réglementation française.

* En France, l'huile de colza est légalement " déconseillée " pour la friture. Au niveau des consommateurs cela entraîne des réactions dont il va falloir tenir compte ;

* la réglementation française ne reconnaît comme AGE que l'acide linoléique ;

- des contraintes de la communication. Pour l'information du consommateur, il convient de distinguer les termes " acide linoléique " et " acide alpha-linolénique »! Exercice difficile pour des mots si proches aux rôles si différents. Par ailleurs, les consommateurs cherchent des margarines à « double usage " pouvant à la fois être tartinées et utilisées en cuisson. Dans ce contexte, ils vont se poser la question de savoir si la margarine contenant du colza n'est pas " dangereuse " pour la santé lors de la cuisson (si l'huile de colza n'est pas recommandée pour la friture, qu'en est-il pour une margarine au colza en cuisson ?).

De plus, la réglementation française ne retenant comme AGE que l'acide linoléique, ceci a une incidence directe sur la communication nutritionnelle dans la mesure où, introduisant du colza dans la formule d'une margarine, on fait logiquement baisser le taux de l'acide linoléique et de ce fait on perd l'appellation « à teneur garantie en AGE ».

Au-delà, I'huile de colza continue d'être perçue comme potentiellement " dangereuse " pour certains consommateurs souvent âgés (qui ont un vague souvenir de l'affaire de l'acide érucique). Enfin, dans le cadre de la problématique OGM et de I'hypersensibilité des consommateurs, l'appellation " nouvelle huile de colza » pose problème car certains consommateurs sont persuadés qu'il s'agit d'un produit renfermant des OGM.

\section{Les trans}

Lorsque l'article du Pr Willett sur les trans est repris par les médias aux États-Unis de façon très alarmiste, les retombées ne tardent pas à arriver en Europe. 
À cette période, en France, les margarines étaient nettement moins riches en trans que leurs équivalents aux États-Unis et la part estimée de trans issus des margarines dans l'apport quotidien se situe à $40 \%$ environ.

Unilever et Astra Calvé pour la France choisissent pourtant de retirer les trans des produits concernés, et depuis 1996 l'ensemble de ses margarines contiennent moins de $1 \%$ de trans (tableau). Dès lors, le dossier pourrait être considéré comme clos, mais c'est sans compter sur les médias. En 1998, une émission sur les trans est diffusée à une heure de grande écoute. Ce reportage date de cinq ans (ce qui n'est pas précisé) et sème le trouble en laissant entendre que les margarines sont nocives. Les consommateurs sont de nouveau inquiets et peu confiants sur les réponses apportées par le Service consommateurs. De plus, pour une clarté d'information totale, Astra-Calvé choisit d'indiquer le taux de trans sur les produits qui ont un étiquetage nutritionnel. Cette démarche n'est pas exigée par la réglementation et va générer des questions inattendues, car les consommateurs assimilent parfois trans à transgéniques, et ne sont pas nécessairement aptes à considérer que inférieur à $1 \%$ (tel qu'indiqué sur l'étiquetage) est un apport quasi nul.

\section{Les phytostérols}

Les maladies cardio-vasculaires représentent $36 \%$ des décès en France et constituent la première cause de mortalité. L'hypercholestérolémie est reconnue comme facteur de risque majeur de ces pathologies.

Les recommandations médicales préconisent dans la prise en charge des patients un régime en première intention afin de mesurer si le patient est réceptif ou non. Ce régime est vécu comme une contrainte et les médecins sautent souvent cette étape pour s'orienter directement vers une prescription de médicaments.

Les phytostérols sont connus depuis les années 50. Pour sa part, le groupe Unilever effectue des recherches depuis une dizaine d'années et a développé une margarine commercialisée dans plusieurs pays et qui attend le feu vert définitif des autorités Novel Foods Europe prochainement.

II s'agit de mettre sur le marché un produit alimentaire qui va renforcer les bénéfices du régime (souvent mal perçu par les patients), un produit quotidien, simple d'usage, et qui va motiver le patient.

C'est une pâte à tartiner à $35 \%$ de matière grasse enrichie en stérols végétaux (apport $8 \%$ ). Une consommation de $20 \mathrm{~g} / \mathrm{j}$ permettra de faire baisser, au bout de 3 semaines en moyenne, le LDL (lipoprotéines de basse densité) - cholestérol de 10 à 14 \% dans le cadre d'un régime adapté.

II s'agira d'une communication spécifique (le produit concerne des patients hypercholestéroléniques) qui va s'adresser en particulier à la population médicale (formation continue des médecins pour la prise en charge du patient par le biais du régime). Un véritable défi pour redonner à l'alimentation la place qui lui revient dans la prise en charge de ce facteur de risque. 


\section{Nos missions d'entreprise}

Il s'agit pour nous de :

* Développer des produits qui répondent aux attentes du consommateur en termes de goût, d'usage et de coûts.

* Accompagner et soutenir les travaux de la recherche en adaptant les formules des produits à l'évolution des données scientifiques.

* S'adapter aux exigences de la réglementation.

* S'appuyer sur un vocabulaire qui, dans le domaine des corps gras, reste très difficile d'accès pour le grand public.

\section{CONCLUSION}

Cette présentation montre la complexité de la communication, mais l'espoir existe aujourd'hui car on assiste à un regroupement des instances en France et à la création possible d'une agence européenne, ce qui va permettre aux industries une simplification des procédures, une expertise indépendante, spécialisée et dédiée, et enfin une évolution de la réglementation.

Enfin, il serait intéressant de réunir un groupe de travail avec des représentants de la recherche scientifique, des pouvoirs publics, des consommateurs, des industriels pour chercher ensemble à redéfinir une terminologie nutritionnelle ayant pour objectif de trouver un langage commun qui clarifie l'information pour le consommateur.

Illustrations 


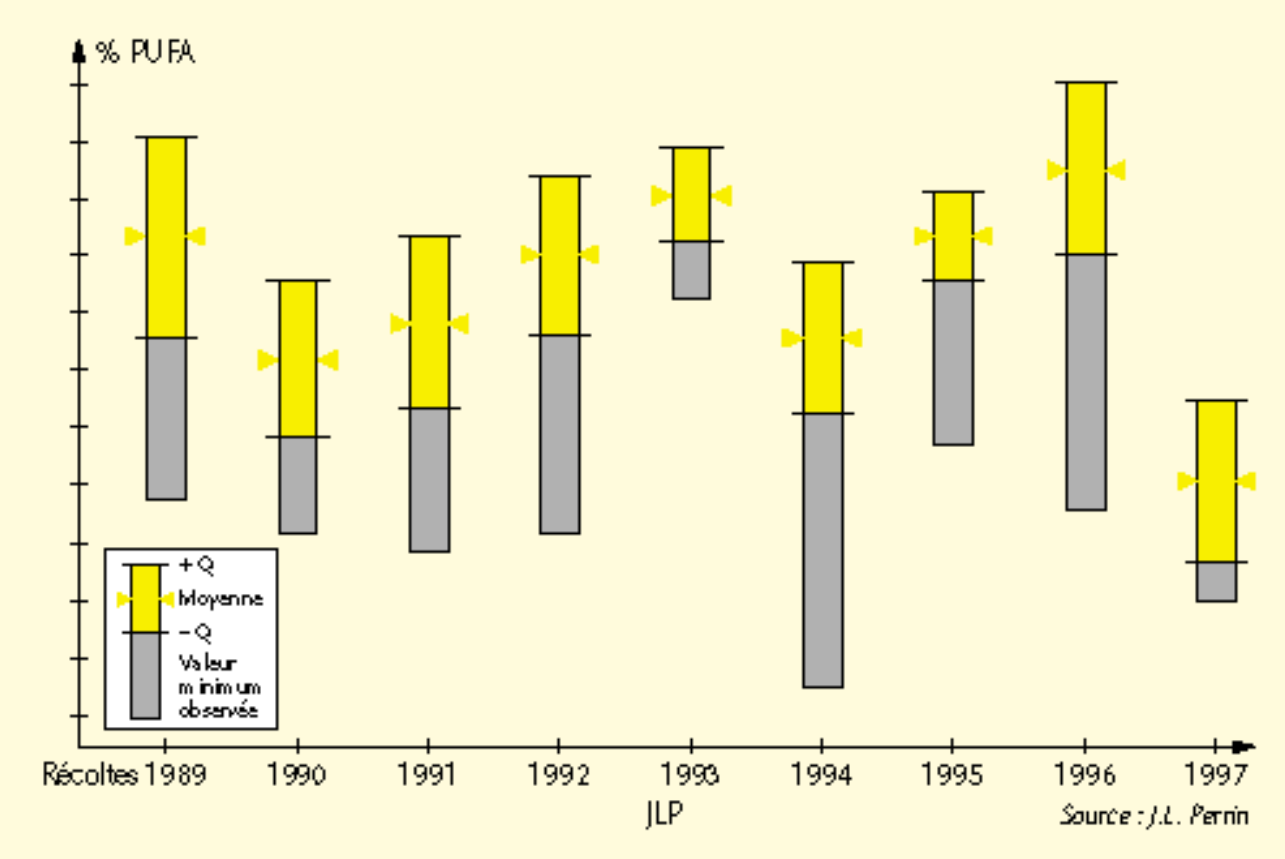

Figure. Variations naturelles de l'apport en linoléique des récoltes de tournesol.

\begin{tabular}{|l|c|c|c|c}
\hline & Septembre 1992 & Août 1994 & Mars 1995 & Mars 1996 \\
\hline FRUT D OR & 8,5 & 0,3 & & \\
\hline PLANTA FIN & 10,0 & & 0,8 & \\
\hline EFFI & 7,0 & & & 0,2 \\
\hline ASTRA & 19,0 & & & 0,6
\end{tabular}

Tableau. Évolutions des teneurs en acides gras trans des margarines (en \%). 